\title{
Attenuation of Pulmonary Mycobacterium Avium Disease by Active Hexose Correlated Compound (AHCC) in Mice
}

Masaki Fujita ${ }^{1 *}$, Takemasa Matsumoto ${ }^{1}$, Ryosuke Hirano¹, Kazunari Ishii ${ }^{2}$, Kenji Hiromatsu², Junji Uchino ${ }^{1}$ and Kentaro Watanabe $^{1}$

${ }^{1}$ Department of Respiratory Medicine, Faculty of Medicine, Fukuoka University, Japan

${ }^{2}$ Department of Microbiology and Immunology, Faculty of Medicine, Fukuoka University, Japan

\begin{abstract}
Introduction: Pulmonary Mycobacterium avium disease is a chronic and progressive disease that is known to be difficult to treat. Active hexose correlated compound (AHCC) is an extract obtained by culturing mycelia of Basidiomycota. AHCC is reported to attenuate several experimental animal infection models. We hypothesized that AHCC could attenuate pulmonary $M$. avium disease in mice.

Methods: Mycobacterium avium $\left(10^{8} \mathrm{cfu} / \mathrm{head}\right)$ was administered intratracheally into mice (C57/BL6). Infected mice were supplied with $1,000 \mathrm{mg} / \mathrm{kg} / \mathrm{day}$ of $\mathrm{AHCC}$ by oral administration until euthanasia. The mice were sacrificed at seven days later or 21 days later after M. avium infection. The lung homogenates were cultured on Middlebrook $7 \mathrm{H} 10$ agar plates for bacterial colony counts. Additionally, the number of inflammatory cells in the lungs was analyzed by FACS. Tissue sections of the lungs were stained by hematoxylin and eosin or Ziehl-Neelsen methods. In addition, the number of bacterial colonies in the macrophages was counted in vitro. Approximately $1 \times 10^{6}$ macrophages were incubated with M. avium at an $\mathrm{MOI}$ of 10 with or without $1 \mathrm{mg} / \mathrm{ml}$ of AHCC.

Results: The administration of AHCC improved lung inflammation caused by $M$. avium according to the histology results and decreased number of $M$. avium in the lungs. In the analysis of lung inflammatory cells, the number of TNFR1 cells and NK cells remained unchanged by AHCC administration, however, the number of TNFR2 cells slightly increased. There was no difference in the number of $M$. avium in macrophages treated with or without AHCC in the in vitro study.
\end{abstract}

Conclusion: AHCC plays a protective role in a murine model of pulmonary M. avium disease.

Keywords: Glucan; Mycobacteriosis; Biological response modifiers; Animal study

\section{Introduction}

Mycobacterium avium is a human pathogen which proliferates intracellularly in phagocytes. The bacterium causes chronic progressive respiratory infection as well as disseminated diseases in immunocompromised hosts such as HIV patients [1,2]. It has been reported that $M$. avium causes a chronic and progressive disease, pulmonary Mycobacterium avium-intracellulare complex (MAC) disease. Pulmonary MAC disease is known to be difficult to treat [3-7]. Therefore, the development of novel treatments for pulmonary MAC disease is needed.

Active hexose correlated compound (AHCC) is an extract obtained by culturing mycelia of Basidiomycota. The main component of AHCC is acetylated $\alpha$-glucan. AHCC has been used as a dietary supplement in various countries. These glucans are thought to provide a carbohydrate that stimulates immune responses [8,9]. It has been previously reported that AHCC influences both natural immunity and acquisition immunity and is also effective against viral infection, Eumycota and other bacteria [10-12]. It has been reported that AHCC attenuated infection caused by Candida albicans, Pseudomonas aeruginosa, and methicillin-resistant Staphylococcus aureus (MRSA) in a neutrophil depleted murine model by cyclophosphamide [13]. Since AHCC itself does not have any antibacterial ability against $C$. albicans, the attenuation effect depends on its immunological effect [14]. AHCC has also been demonstrated to have a protective effect against Klebsiella pneumoniae infection. AHCC could improve the immune defect induced by injury, infection or starvation [15]. Recent studies demonstrated that the activity of natural killer (NK) cells was improved by AHCC in an influenza virus model $[16,17]$. AHCC is thus considered to play a role as a biological response modifier (BRM).
We hypothesized that AHCC could act as a BRM and attenuate pulmonary $M$. avium disease. This study aimed to investigate the beneficial effect of AHCC in M. avium infection.

\section{Materials and Methods}

\section{Bacterial strain}

The clinically isolated M. avium strain FN from our hospital was used in this study. All isolates were grown in Middlebrook 7H9 broth with Middlebrook ADC enrichment (Becton Dickinson and Company, Sparks Glencoe, MD) at $37^{\circ} \mathrm{C}$ with shaking or on Middlebrook $7 \mathrm{H} 10$ agar with Middlebrook OADC enrichment (Becton Dickinson and Company) at $37^{\circ} \mathrm{C}$ for 14 days. The plates were incubated at $37^{\circ} \mathrm{C}$ in $90 \%$ humidity and the colonies were counted after 14 days $[18,19]$.

\section{Animal model of M. avium infection}

C57BL/6 mice of the same age, three months, were used. The intratracheal administration of $M$. avium $\left(1 \times 10^{8} \mathrm{CFU} / \mathrm{head}\right)$ in 50 $\mu \mathrm{l}$ of sterile saline was performed via tracheotomy to the mice under

*Corresponding author: Masaki Fujita, Department of Respiratory Medicine Faculty of Medicine, Fukuoka University, Fukuoka 814-0180, Japan, Tel: 81-92 801-1011; Fax: 81-92-865-6220; E-mail: mfujita@fukuoka-u.ac.jp

Received October 09, 2015; Accepted October 28, 2015; Published November 02, 2015

Citation: Fujita M, Matsumoto T, Hirano R, Ishii K, Hiromatsu K, et al (2015) Attenuation of Pulmonary Mycobacterium Avium Disease by Active Hexose Correlated Compound (AHCC) in Mice. J Nutr Disorders Ther 5: 174 doi:10.4172/2161- 0509.1000174

Copyright: (c) 2015 Fujita M, et al. This is an open-access article distributed under the terms of the Creative Commons Attribution License, which permits unrestricted use, distribution, and reproduction in any medium, provided the original author and source are credited. 
anaesthesia [20]. AHCC was provided from Amino Up Chemical (Sapporo, Japan). Detail of AHCC was described previously [8-17]. Infected mice were supplied with $1,000 \mathrm{mg} / \mathrm{kg} /$ day of AHCC by oral administration until euthanasia. This dose of AHCC has been used previously and does not produce toxic effects in young mice [21]. AHCC was added to the drinking water [20] so that the dose is rough assessment doses. Infection with water (water group) and a noninfection control mice group were also set up. Mice were sacrificed at seven days after infection or 21 days after $M$. avium administration. The lung homogenates were cultured on Middlebrook $7 \mathrm{H} 10$ agar plates to determine the colony count. After sacrifice, the lungs were fixed with $10 \%$ formalin for 24 hours and embedded in paraffin. Tissue sections of the lungs were stained with hematoxylin and eosin (H-E) and ZiehlNeelsen (Z-N) staining.

BAL was performed on day 21 after intratracheal $M$. avium instillation. In the mice with a tracheal tube inserted, the lungs were lavaged with $0.5 \mathrm{ml}$ aliquots of phosphate-buffered saline ten times for a total of $5 \mathrm{ml}$. The total cell counts were determined with a hemocytometer. Differential counts of BAL fluid were performed on 200 cells from smears stained with a modified Wright's stain (DiffQuik; American Scientific Products, McGas Park, IL).

This study was approved by our Institutional Animal Care and Use Committee (approved number; 0911352 by Fukuoka University).

\section{Flow cytometric analysis (FACS)}

Inflammatory cells in the lungs were also analyzed by FACS using the lung homogenates. After sacrifice, the individual lungs were dissociated in $5 \mathrm{ml}$ PBS containing Collagenase II $(2.5 \mathrm{mg} / \mathrm{ml}$, SigmaAldrich. St. Louis, $\mathrm{MO}$ ) using the mouse lung dissociation program 1 on a GentleMACS tissue dissociator (Miltenyi Biotec Ltd., DE). This was followed by incubation in an incubator $\left(37^{\circ} \mathrm{C}, 30 \mathrm{~min}\right)$ and a final GentleMACS dissociation using the mouse lung dissociation program 2. Cells were pelleted by centrifugation at $300 \mathrm{~g}$ for $10 \mathrm{~min}$ at $4^{\circ} \mathrm{C}$. Pellets were washing twice with PBS, and lung mononuclear cells were purified by discontinuous $45 / 67.5 \%$ Percoll gradient centrifugation. Cells were dissolved with 45\% Percoll (GE Healthcare UK Ltd, UK) and overlay the $45 \%$ Percoll suspension onto $67.5 \%$ Percoll centrifuged at $1000 \mathrm{~g}$ for $30 \mathrm{~min}$. Cells from the $45 / 67.5 \%$ interface were collected, washed, and resuspended in RPMI media. These cells were staining for analysis. Before staining, anti-CD16/32 antibody (clone 93; BioLegend, San Diego, CA) were added to the cells for block nonspecific staining and cells were incubated for $20 \mathrm{~min}$ at $4^{\circ} \mathrm{C}$. Cells were stained with FITC-conjugated anti-NK mAb (clone DX5; eBioscience, San Diego, CA), APC-conjugated anti-CD120a mAb (clone55R-286; BioLegend) and PE-conjugated anti-CD120b mAb (cloneTR75-89; BioLegend) at $4^{\circ} \mathrm{C}$ for $30 \mathrm{~min}$. After washing twice with PBS, cells were collected on a FACSCanto II (BD Biosciences, San Jose, CA) and data from 15,000 to 20000 individual cells were analyzed using BD FACSDiva software (BD Biosciences).

\section{Macrophage isolation and infection by M. avium}

Bacterial proliferation in murine macrophages was assayed by methods previously described elsewhere [19,22,23] with some modifications. Briefly, the peritoneal macrophages were obtained at three days after inoculation in $2 \mathrm{ml}$ of 3\% thioglycollate medium (Becton Dickinson and Company, Franklin Lakes, NJ).

The macrophages were incubated in RPMI1640 medium with 10\% heat-inactivated fetal bovine serum (Sigma-Aldrich Japan, Tokyo, Japan) without antibiotics using 12-well cell culture plates (Becton
Dickinson and Company, Tokyo, Japan). RAW cells were also used; RAW cells were incubated with DMEM medium with $10 \%$ heatinactivated fetal bovine serum without antibiotics. Approximately $1 \mathrm{x}$ $10^{6}$ cells were incubated per well and M. avium was added to the culture medium at a MOI of 10 . AHCC $(1 \mathrm{mg} / \mathrm{ml})$ was subsequently added. After culturing, the macrophages were collected on day 1, day 2, and day 4 . Collected macrophages were washed twice by PBS and lysed by the addition of sterilized water. The lysed medium was then plated onto Middlebrook 7H10 agar plates and the bacterial number was counted after two weeks of incubation at $37^{\circ} \mathrm{C}$.

\section{Statistical Analysis}

The data are expressed as the mean + standard error (SE). The Mann-Whitney test was used to compare the two groups. A value of $\mathrm{p}<0.05$ was considered to indicate a significant difference. All statistical analyses were performed using the StatView version 5.0 software program.

\section{Results}

First, we evaluated the safety of AHCC administration in mice. There was no apparent pathological change or change in the body weight after the administration of $1,000 \mathrm{mg} / \mathrm{kg} / \mathrm{day}$ of AHCC for 21 days (data not shown). Then, M. avium-inoculated mice were supplied with $1,000 \mathrm{mg} / \mathrm{kg} /$ day of AHCC by oral administration until euthanasia. M. avium-inoculated mice administered water was also set up as the control group. In a previous study, doxycycline was successfully supplied in the water in the same manner [20]. The dose of AHCC was determined by previous reports [17]. Body weight was measured during AHCC administration. There was no apparent difference between AHCC treated mice and the controls (Figure 1). The lung histology was then evaluated. M. avium-inoculation resulted in the infiltration of inflammatory cells around the bronchus after 21 days, and AHCC treatment appeared to attenuate inflammatory cell infiltration (Figure 2). The colony number was also evaluated. AHCC treatment decreased the colony count of $M$. avium (Figure 3 ). The administration of AHCC attenuated lung inflammation caused by $M$. avium according to the histology results and decreased colony count of $M$. avium in the lungs.

We next attempted to determine the mechanism of AHCC responsible for the attenuation of $M$. avium infection. First, we performed an in vitro study. AHCC was added to macrophages infected with M. avium. As shown in (Figure 4), there was no difference

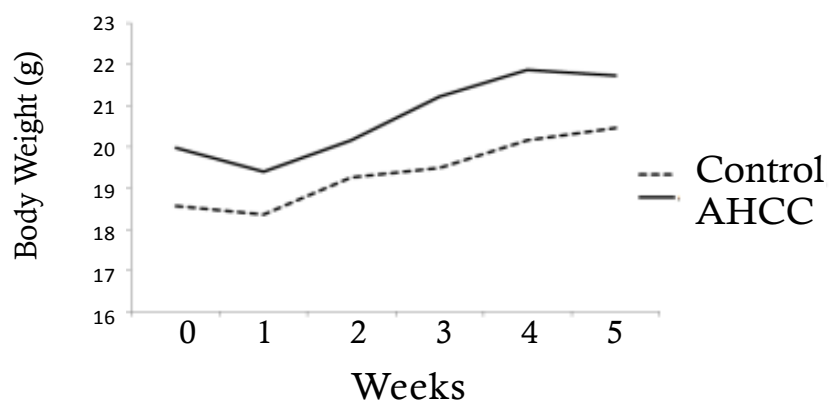

Figure 1: The body weight of mice infected with $M$. avium infection. The body weight was measured weekly after $M$. avium infection. Dashed line, M. avium-inoculated mice supplied with water; Line, M. avium-inoculated mice supplied with AHCC. Each group consisted of four mice. There were no significant differences between the two groups. 

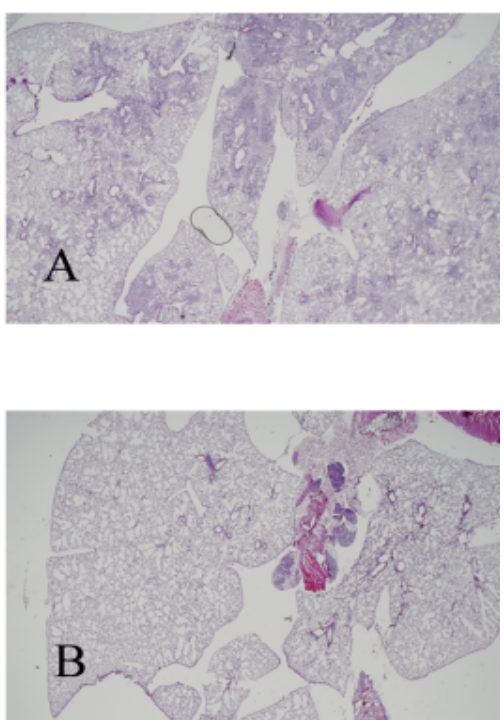

Figure 2: The histologic assessment of the lung after $M$. avium inoculation. (A) The lung from a M. avium-inoculated mouse supplied with water. (B) The lung tissue from a $M$. avium-inoculated mouse supplied with AHCC (H-E staining, original magnification: 20x).

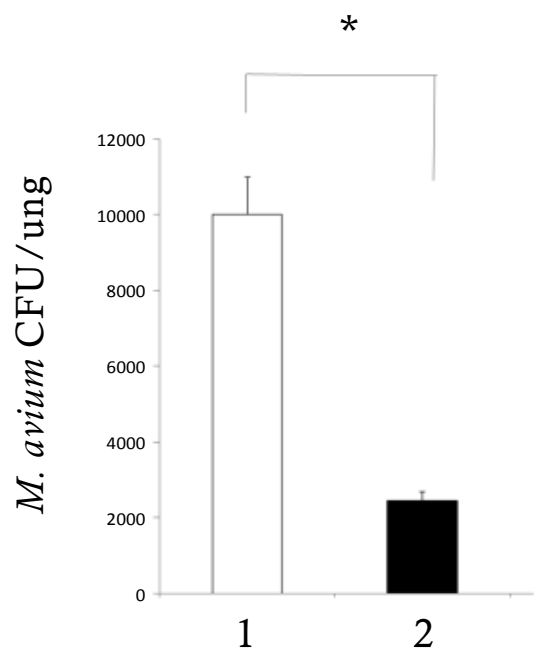

Figure 3: The bacterial growth in the lung. Lane 1, M. avium-inoculated mice supplied with water; lane 2, M. avium-inoculated mice supplied with AHCC. Each group consisted of four mice. * indicates significant differences between two groups.

in the colony count of $M$. avium in RAW macrophages treated with or without AHCC in vitro. The study using peritoneal macrophages demonstrated similar results (data not shown). Lung inflammatory cells were subsequently analyzed by FACS. As a result, the number of TNFR1 and NK cells (Figure 5) remained unchanged following AHCC administration, however, the number of TNFR2 cells slightly increased. Interestingly, the number of BAL fluid cells, mainly macrophages, tended to be increased, although this increase was not significant (Figure 6).

\section{Discussion}

In this study, we demonstrated the attenuation effect of AHCC in $M$. avium infection in mice. AHCC has been reported to show

a protective role against Candida, influenza virus, and Klebsiella infection. AHCC influences both the natural immunity and acquisition immunity $[13,15,16]$. Because an in vitro study did not demonstrate bactericidal activity, the effect of AHCC appears to depend on its role

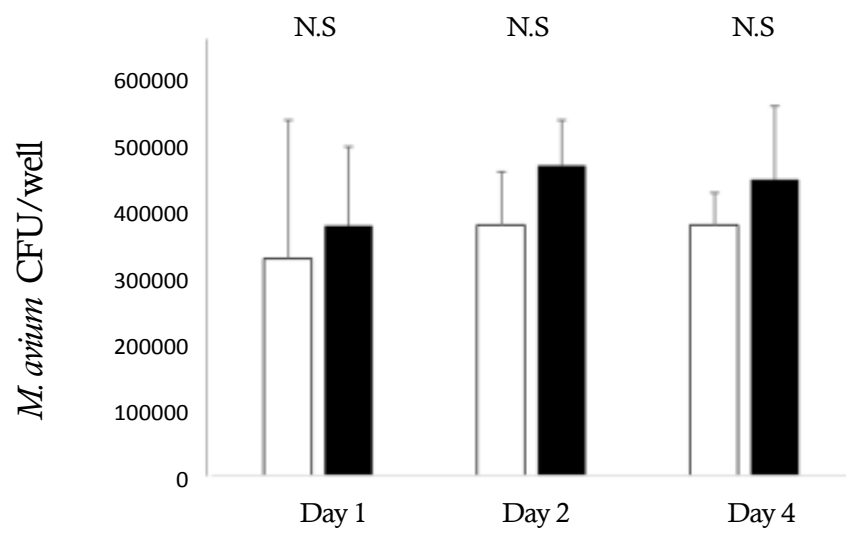

Figure 4: The bacterial growth in the macrophages. Lane 1, M. aviuminoculated mice supplied with water; lane 2; M. avium-inoculated mice supplied with AHCC. There were no significant differences (N.S.) between the two groups.

A

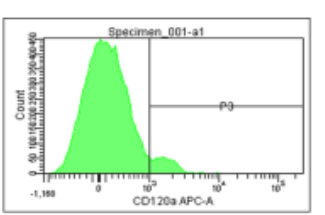

TNFRI

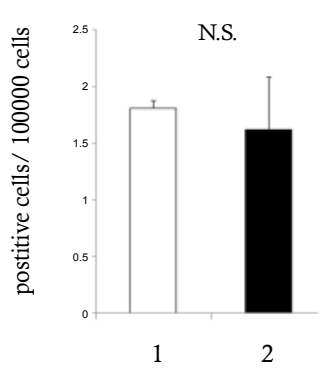

B NK cell

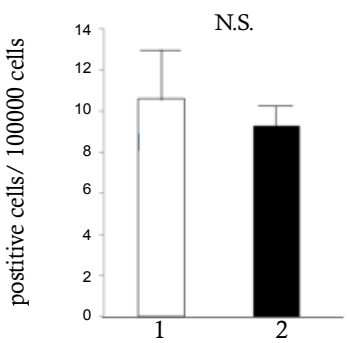

Figure 5: An analysis of the lung inflammatory cells by FACS. (A) Images of FACS for TNFRI and II antibodies. Lane 1, M. avium-inoculated mice supplied with water; lane 2, M. avium-inoculated mice supplied with AHCC. (B) An analysis of the lung inflammatory cells for NK antibody. Lane 1, M. avium-inoculated mice supplied with water; lane 2, M. avium-inoculated mice supplied with AHCC. There were no significant differences (N.S.) between the two groups. 

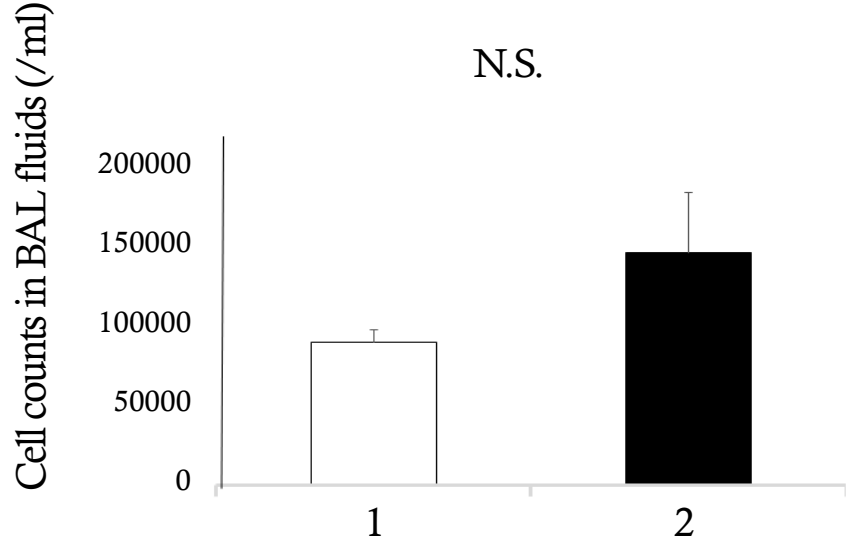

Figure 6: The inflammatory cell accumulation in BAL fluids. Lane 1, M. avium-inoculated mice supplied with water; lane 2, M. avium-inoculated mice supplied with AHCC. An inhibition of inflammatory cytokines was observed, however, it was not significant. Each group consisted of four mice. N.S. indicates no significant differences.

as a BRM. In fact, AHCC treatment tended to increase the number of macrophages in BAL fluids.

M. avium is an intracellular pathogen that grows in macrophage phagolysosomes. Th1 immune responses are thought to be important in protection against intracellular pathogens [24,25]. Specifically, the involvement of IFN- $\gamma$ and IL-12 has been demonstrated in the protection against $M$. avium infection because patients with defects in IFN- $\gamma /$ IL-12 signaling are more susceptible to disseminated and extrapulmonary M. avium diseases [26,27]. Additionally, the TNF signal is involved in immunoprotection. TNFR1 knockout mice are known to be susceptible to M. avium [28]. In this study, we measured the number of TNFR1-positive cells in the lung, which remained unchanged after AHCC treatment. We found a slight increase in the number of TNFR2 cells, however, the role of TNFR2 is unknown. A recent report revealed a novel role for NK cells, perforin, and granulysin in killing Mycobacterium and highlights a potential alternative defense mechanism that the immune system can use against mycobacterial infection [29]. AHCC has been previously reported to increase the number of NK cells in aged mice. $\alpha$-1,4-glucans are recognized by C-type lectins, such as Dectin-1 on NK cells, thus initiating innate immunity [30]. The mechanism of attenuation was thought to be the activation of NK cells [17]. However, the number of NK cells remained unchanged in this study. The attenuation mechanism should be investigated in future studies.

In the present study, AHCC demonstrated a protective role against $M$. avium infection. However, the mechanism remains unclear. Recently, AHCC has been shown to play a potential role in the orchestration of immune responses and the maintenance of immune homeostasis, in part by priming the TLR- 2 and TLR- 4 gate at the intestinal epithelium. Such a response is likely due to the recognition of non-pathogenic food-associated molecular patterns (FAMPs) such as those found to be associated with other mushroom or yeast-derived compounds [31]. Further studies of the mechanism by which AHCC affects immunoprotection are required.

\section{Acknowledgements}

We would like to express our gratitude to Amino Up Chemical for providing AHCC. We appreciate the help of Dr. Brian Quinn for his assistance in preparing the manuscript.

\section{Conflict of Interest}

The authors declare no conflicts of interest.

\section{References}

1. Medical Section of the American Lung Association (1997) Diagnosis and treatment of disease caused by nontuberculous mycobacteria. Am J Respir Crit Care Med 156: S1-25.

2. Griffith DE, Aksamit T, Brown-Elliott BA, Catanzaro A, Daley C, et al; ATS Mycobacterial Diseases Subcommittee; American Thoracic Society; Infectious Disease Society of America (2007) An official ATS/IDSA statement: diagnosis, treatment, and prevention of nontuberculous mycobacterial diseases. Am J Respir Crit Care Med 175: 367-416.

3. Wallace RJ Jr, Brown BA, Griffith DE, Girard WM, Murphy DT (1996) Clarithromycin regimens for pulmonary Mycobacterium avium complex: the first 50 patients. Am J Respir Crit Care Med 153: 1766-1772.

4. Griffith DE, Brown BA, Girard WM, Griffith BE, Couch LA, et al (2001) Azithromycin-containing regimens for treatment of Mycobacterium avium complex lung disease. Clin Infect Dis 32: 1547-1553.

5. Tanaka E, Kimoto T, Tsuyuguchi K, Watanabe I, Matsumoto H, et al (1999) Effect of clarithromycin regimen for Mycobacteriuim avium complex pulmonary disease. Am J Respir Crit Care Med 160: 866-872.

6. Corpe RF (1981) Surgical management of pulmonary disease due to Mycobacterium avium-intracellulare. Rev Infect Dis 3: 1064-1067.

7. Moran JF, Alexander LG, Stauh EW, Young WG, Sealy WC (1983) Long-term results of pulmonary resection for atypical mycobacterial disease. Am Thorac Surg 35: 597-604.

8. Spierings EL, Fujii H, Sun B, Walshe T (2007) A Phase I study of the safety of the nutritional supplement, active hexose correlated compound, AHCC, in healthy volunteers. J Nutr Sci Vitaminol 53: 536-539.

9. Terakawa N, Matsui Y, Satoi S, Yanagimoto H, Takahashi K, et al (2008) Immunological effect of active hexose correlated compound (AHCC) in healthy volunteers: a double-blind, placebo-controlled trial. Nutr Cancer 60: 643-651.

10. Nogusa S, Gerbino J, Ritz BW (2009) Low-dose supplementation with active hexose correlated compoundimproves the immune response to acute influenza infection in C57BL/6 mice. Nutr Res 29: 139-143.

11. Aviles H, O'Donnell P, Orshal J, Fujii H, Sun B, et al (2008) Active hexose correlated compound activates immune function to decrease bacterial load in a murine model of intramuscular infection. Am J Surg 195: 537-545.

12. Fujii H, Nishioka H, Wakame K, Sun B (2007) Nutritional food active hexose correlated compound (AHCC) enhances resistance against bird flu. Jap $J$ Complement Altern Med 4: 37-40.

13. Ishibashi H, lkeda T, Tansho S, Ono Y, Yamazaki M, et al (2000) Prophylactic efficacy of a basidiomycetes preparation AHCC against lethal opportunistic infections in mice. Yakugaku Zasshi 120: 715-719.

14. Ikeda T, Ishibashi H, Fujisaki R, Yamazaki M, Wakame K, et al (2003) Prophylactic efficacy of a basidiomycetes preparation AHCC against lethal Candida albicans infection in experimental granulocytopenic mice. Nihon Ishinkin Gakkai Zasshi 44: 127-131.

15. Aviles H, Belay T, Fountain K, Vance M, Sun B, et al (2003) AHCC enhances resistance to Klebsiella pneumniae infection in mice in the hindlinb-unloading model of spaceflight conditions. J Appl Physiol 95: 491-496.

16. Ritz BW, Nogusa S, Ackerman EA, Gardner EM (2006) Supplementation with Active Hexose Correlated Compound Increases the Innate Immune Response of Young Mice to Primary Influenza Infection. J Nutr 136: 2868-2873.

17. Ritz BW (2008) Supplementation with active hexose correlated compound increases survival following infectious challenge in mice. Nutr Rev 66: 526-531.

18. Fratazzi C, Manjunath N, Arbeit RD, Carini C, Gerken TA, et al (2000) A macrophage invasion mechanism for mycobacteria implicating the extracellular domain of CD43. J Exp Med 192: 183-192.

19. Fujita M, Harada E, Matsumoto T, Mizuta Y, Ikegame S, et al (2010) Impaired host defence against Mycobacterium avium in mice with chronic granulomatous disease. Clin Exp Immunol 160: 457-460.

20. Fujita M, Ye Q, Ouchi H, Harada E, Inoshima I, et al (2006) Doxycycline attenuated pulmonary fibrosis induced by bleomycin in mice. Antimicrob Agents Chemother 50: 739-743. 
Citation: Fujita M, Matsumoto T, Hirano R, Ishii K, Hiromatsu K, et al. (2015) Attenuation of Pulmonary Mycobacterium Avium Disease by Active Hexose Correlated Compound (AHCC) in Mice. J Nutr Disorders Ther 5: 174. doi:10.4172/2161- 0509.1000174

21. Shigama K, Nakaya A, Wakame K, Nishioka H, Fujii H (2009) Alleviating effect of active hexose correlated compound (AHCC) for anticancer drug-induced side effects in non-tumor-bearing mice. J Exp Ther Oncol 8: 43-51.

22. Yoshida S, Goto Y, Mizuguchi Y, Nomoto K, Skamene E (1991) Genetic control of natural resistance in mouse macrophages regulating intracellular Legionella pneumophila multiplication in vitro. Infect Immun 59: 428-432.

23. Fujita M, Ikegame S, Harada E, Ouchi H, Inoshima I, et al (2008) TNF receptor 1 and 2 contribute in different ways to resistance to Legionella pneumophilainduced mortality in mice. Cytokine 44: 298-303.

24. Trinchieri G (1997) Cytokines acting on or secreted by macrophages during intracellular infection (IL-10, IL-12, IFN-y). Curr Opin Immunol 9: 17-23.

25. Méndez-Samperio $P$ (2010) Role of interleukin-12 family cytokines in the cellular response to mycobacterial disease. Int J Infect Dis 14: e366-e371.

26. Altare F, Durandy A, Lammas D, Emile JF, Lamhamedi S, et al (1998) Impairment of mycobacterial immunity in human interleukin-12 receptor deficiency. Science 280: 1432-1435
27. Patel SY, Ding L, Brown MR, Lantz L, Gay T, et al (2005) Anti-IFN-Y autoantibodies in disseminated nontuberculous mycobacterial infections. J Immunol 175: 4769-4776.

28. Tashiro N, Fujita M, Matsumoto T, Hirano R, Uchino J, et al (2015) Doxycycline attenuated Mycobacterium avium induced inflammation in mice. Mycobacterial Diseases.

29. Lu CC, Wu TS, Hsu YJ, Chang CJ, Lin CS, et al (2014) NK cells kill mycobacteria directly by releasing perforin and granulysin. J Leukoc Biol 96: 1119-1129.

30. Nguyen KB, Salazar-Mather TP, Dalod MY, Van Deusen JB, Wei XQ, et al (2002) Coordinated and distinct roles for IFN- $\alpha \beta$, IL-12, and IL-15 regulation of NK cell responses to viral infection. J Immunol 169: 4279-4287.

31. Mallet JF, Graham É, Ritz BW, Homma K, Matar C (2015) Active Hexose Correlated Compound (AHCC) promotes an intestinal immune response in $\mathrm{BALB} / \mathrm{c}$ mice and in primary intestinal epithelial cell culture involving toll-like receptors TLR-2 and TLR-4. Eur J Nutr. 